\title{
EXTEND TIME COULD LEAD TO REDUCE IT!
}

\section{Boithias 1,7, C Boissinot ${ }^{2}$, S Le Foulgoc 3 , N Lodé4, A Tasseau ${ }^{5}$, G Jourdain 6,7}

1 Pediatric intensive care unit and neonatal medicine. South Paris University Hospitals, medical center Bicetre. France

2 Maternity unit. Robert Debré Hospital. Paris.France

3 Neonatal intensive care unit. South Francilien Hospital. Corbeil. France

4 Mobile pediatric intensive care unit (SAMU 75). Robert Debré Hospital. Paris. France

5 Neonatology unit. Saint Joseph Hospital. Paris. France

6 Mobile pediatric intensive care unit (SAMU 92). South Paris University Hospitals, medical center A Beclere France

7 Medical simulation center LabForSims, South Paris University. France

\section{Background and aims}

ILCOR publishes guidelines for newborn resuscitation and recommends proceeding in a step-by-step manner. Positive pressure ventilation (PPV) (step B) should be initiated if the infant is not breathing or if the heart rate is less than $100 \mathrm{bpm}$ after step A has been completed. In the 2015 recommendations time to complete step A was extended.

Do the new guidelines of ILCOR change the execution of steps A and B in simulated newborn resuscitation scenarios?

\section{Methods}

Training sessions are part of the mandatory teaching of newborn resuscitation for first-year pediatric residents in the entire Paris region. The same educational progression was maintained in all the sessions.

The training sessions were separated into two periods, before and after 2015 guidelines. We included all the scenarios which required PPV after completion of step A. We excluded the scenarios without required PPV. We defined nine, step A and step B required tasks. Video footage of all scenarios were reviewed by two certified instructors.

\section{Results}

For the study, 336 residents completed a total of 184 scenarios during the simulation-based training; 157 students completed 85 scenarios in period 1, and 179 students completed 95 scenarios in period 2. We included 124 scenarios, 48 in the first period and 72 in the second period. All residents participated in at least one scenario. Results are summed up in tables .

Time of PPV start during sessions in both two periods
(seconds))

Period 1

Period 2

\begin{tabular}{llll} 
& Period 1 & Period 2 & $\mathrm{p}$ (anova) \\
& $\mathrm{N}=48$ & $\mathrm{~N}=72$ & \\
$\begin{array}{l}\text { Time of PPV start } \\
\text { (second) }\end{array}$ & $58.08 \pm 15.9$ & $57 \pm 14$ & $\mathrm{NS}$ \\
$\begin{array}{l}\text { Tasks executed } \\
\text { within 1 minute } \\
(\mathrm{n}=9)\end{array}$ & $7.58 \pm 1.02$ & $8.09 \pm 0.77$ & $\mathrm{p}=0.0022$ \\
& & & \\
\hline
\end{tabular}

$\begin{array}{llll}\text { Scenario } 2 & \text { Scenario } 4 & \text { Scenario } 5 & \mathrm{p} \\ 61 \pm 18 & 56.8 \pm 15 & 54.8 \pm 14 & \mathrm{NS} \\ 63 \pm 9 & 53 \pm 11 & 50.7 \pm 9 & \mathrm{P}=0.0049\end{array}$

\section{Conclusions}

PPV start was not significantly different in the two periods. Completion of tasks was better in period 2. We hypothesize that less time pressure leads to better results and doesn't delay PPV. 